University of Wollongong

Research Online

$1-1-2017$

\title{
Using a logic model to evaluate the Kids Together early education inclusion program for children with disabilities and additional needs
}

\author{
Kathleen F. Clapham \\ University of Wollongong, kclapham@uow.edu.au \\ Claire E. Manning \\ University of Wollongong, claire@magnolialearning.com.au \\ Kate Williams \\ University of Wollongong, kathrynw@uow.edu.au \\ Ginger O'Brien \\ Noah's Shoalhaven \\ Margaret Sutherland \\ Noah's Shoalhaven
}

Follow this and additional works at: https://ro.uow.edu.au/ahsri

Research Online is the open access institutional repository for the University of Wollongong. For further information contact the UOW Library: research-pubs@uow.edu.au 


\title{
Using a logic model to evaluate the Kids Together early education inclusion program for children with disabilities and additional needs
}

\author{
Abstract \\ Despite clear evidence that learning and social opportunities for children with disabilities and special \\ needs are more effective in inclusive not segregated settings, there are few known effective inclusion \\ programs available to children with disabilities, their families or teachers in the early years within \\ Australia. The Kids Together program was developed to support children with disabilities/additional \\ needs aged $0 \_8$ years attending mainstream early learning environments. Using a key worker \\ transdisciplinary team model, the program aligns with the individualised package approach of the \\ National Disability Insurance Scheme (NDIS). Aim This paper reports on the use of a logic model to \\ underpin the process, outcomes and impact evaluation of the Kids Together program. Methods The \\ research team worked across 15 Early Childhood Education and Care (ECEC) centres and in home and \\ community settings. A realist evaluation using mixed methods was undertaken to understand what \\ works, for whom and in what contexts. The development of a logic model provided a structured way to \\ explore how the program was implemented and achieved short, medium and long term outcomes within a \\ complex community setting. Discussion and conclusion Kids Together was shown to be a highly effective \\ and innovative model for supporting the inclusion of children with disabilities/additional needs in a range \\ of environments central for early childhood learning and development. The use of a logic model provided \\ a visual representation of the Kids Together model and its component parts and enabled a theory of \\ change to be inferred, showing how a coordinated and collaborative approached can work across multiple \\ environments.

\section{Publication Details} \\ K. Clapham, C. Manning, K. Williams, G. O'Brien \& M. Sutherland, "Using a logic model to evaluate the Kids \\ Together early education inclusion program for children with disabilities and additional needs", Evaluation \\ and Program Planning 61 (2017) 96-105.
}




\section{Accepted Manuscript}

Title: Using a logic model to evaluate the Kids Together early education inclusion program for children with disabilities and additional needs

Author: Kathleen Clapham Claire Manning Kathryn Williams Ginger O'Brien Margaret Sutherland

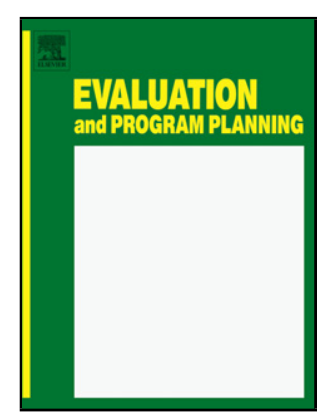

PII:

DOI:

Reference:

S0149-7189(16)30133-1

http://dx.doi.org/doi:10.1016/j.evalprogplan.2016.12.004

EPP 1400

To appear in:

Received date: 24-6-2016

Revised date: 28-11-2016

Accepted date: $\quad$ 7-12-2016

Please cite this article as: Clapham, Kathleen., Manning, Claire., Williams, Kathryn., O'Brien, Ginger., \& Sutherland, Margaret., Using a logic model to evaluate the Kids Together early education inclusion program for children with disabilities and additional needs.Evaluation and Program Planning http://dx.doi.org/10.1016/j.evalprogplan.2016.12.004

This is a PDF file of an unedited manuscript that has been accepted for publication. As a service to our customers we are providing this early version of the manuscript. The manuscript will undergo copyediting, typesetting, and review of the resulting proof before it is published in its final form. Please note that during the production process errors may be discovered which could affect the content, and all legal disclaimers that apply to the journal pertain. 


\section{Title}

Using a logic model to evaluate the Kids Together early education inclusion program for children with disabilities and additional needs

Kathleen Clapham ${ }^{1}$, Claire Manning ${ }^{1}$, Kathryn Williams ${ }^{1}$, Ginger O’Brien ${ }^{2}$ and Margaret Sutherland ${ }^{2}$

${ }^{1}$ University of Wollongong, Centre for Health Services Development (CHSD), Australian Health Services Research Institute (AHSRI), Wollongong, 2522, Australia ${ }^{2}$ Noah's Shoalhaven, Nowra, 2541, Australia

\section{Corresponding Author}

Professor Kathleen Clapham

Australian Health Services Research Institute

kclapham@uow.edu.au

Phone: +61 (2) 42215171

\section{Highlights}

- Effective innovative model supporting inclusion in early childhood education.

- Program aligns well with the National Disability Insurance Scheme (NDIS)

- Logic model demonstrates how outcomes are achieved in a complex community setting.

- Key worker approach simplifies the coordination of therapies.

- Children benefit from opportunities to practise goals across multiple environments. 


\section{Abstract}

Despite clear evidence that learning and social opportunities for children with disabilities and special needs are more effective in inclusive not segregated settings, there are few known effective inclusion programs available to children with disabilities, their families or teachers in the early years within Australia. The Kids Together program was developed to support children with disabilities/additional needs aged 0-8 years attending mainstream early learning environments. Using a key worker transdisciplinary team model, the program aligns with the individualised package approach of the National Disability Insurance Scheme (NDIS).

Aim: This paper reports on the use of a logic model to underpin the process, outcomes and impact evaluation of the Kids Together program.

Methods: The research team worked across 15 Early Childhood Education and Care (ECEC) centres and in home and community settings. A realist evaluation using mixed methods was undertaken to understand what works, for whom and in what contexts. The development of a logic model provided a structured way to explore how the program was implemented and achieved short, medium and long term outcomes within a complex community setting.

Discussion and conclusion: Kids Together was shown to be a highly effective and innovative model for supporting the inclusion of children with disabilities/additional needs in a range of environments central for early childhood learning and development. The use of a logic model provided a visual representation of the Kids Together model and its component parts and enabled a theory of change to be inferred, showing how a coordinated and collaborative approached can work across multiple environments.

\section{Key Words}

Program evaluation; early childhood; inclusion; logic model; disabilities; National Disability Insurance Scheme; NDIS. 


\section{Introduction and Background}

The inclusion of children with disabilities in early childhood education is a relatively new practice within Australia, despite the growing evidence that developmental gains from early intervention for children with disabilities is as good or better in inclusive settings as opposed to segregated, specialist services (Odom 2000, Odom 2011, Underwood, Valeo et al. 2012, Tanner, Cook et al. 2013, Lee 2015, Sainato 2015). Intensive, tailored interventions provided effectively in mainstream settings have benefits for the children's social development and the wellbeing of their families. However, there are few rigorously evaluated inclusion programs in early childhood education settings in Australia (Dew, Bortoli et al. 2014, Bortolli and Bundy 2015).

Kids Together was designed to support children with disabilities/ additional needs, aged 0-8 years, attending mainstream early learning environments. The model represents a significant change to the way therapists and children's specialists deliver services to children with disabilities. Using a key worker transdisciplinary team approach, key workers are supported by colleagues from allied health and educational professional backgrounds, working with children with disabilities within Early Childhood Education and Care (ECEC) centres, at home and in the community. The key worker transdisciplinary teamwork approach has grown in popularity across a range of healthcare services in recent years. It has been shown to have the advantage of supporting families in an efficient way by providing a team of professionals, led by a main key worker, working collaboratively to evaluate, plan and investigate appropriate services and programs (Moore 2012).

The model is also consistent with an individualised package approach to disabilities support provided by the National Disability Insurance Scheme (NDIS), a major reform in the way 
people with disabilities are supported in Australia (National Disability Insurance Scheme 2014). The move from program based funding to individualised support packages promises new opportunities, but also poses important challenges for service providers in the disabilities services sector which have been highlighted in recent research from the NDIS trial site (Howard, Blakemore et al. 2015). Howard et al (2015) for example describe "a parade of different services" in the ECECs in the NDIS Hunter trial and report parents' distress at having continuous staff changes or different professionals providing components of support in isolation. Two other significant challenges with the NDIS changes include assumptions about the capacity of parents and families to support their children through this new approach and equity of access to appropriate services, particularly in regional and rural settings (Howard, Blakemore et al. 2015).

This paper presents the results of the evaluation of Kids Together as it was implemented for the first time across 15 sites in the Illawarra region of New South Wales, in the lead up to the rollout of the National Disability Insurance Scheme (NDIS). A realist evaluation was undertaken to test the effectiveness of Kids Together in this context and to assess its suitability for future expansion across a broader range of settings (Pawson and Tilley 1997). The overall goal was to bring together lessons learnt in the delivery of the program and to capture evidence of its impact and effectiveness from all project sites. The evaluation aimed to assess what had been achieved, whether it made a difference and why, and to understand the processes by which any changes had occurred and how the program might be expanded to other areas and contexts. This paper focuses on the development and core components of the Kids Together program, highlights the importance of the logic model in undertaking a collaborative realist evaluation and reports on the findings. A more comprehensive and detailed account of the evaluation is reported in elsewhere (Author and Author 2015). 


\section{Methods}

The evaluation of Kids Together employed a realist approach. Derived from the work of Pawson and Tilley (Pawson and Tilley 1997, Pawson and Tilley 2004), realist evaluation is a theory driven method that assumes that programs 'work' in different ways for different people and that the context in which a program operates must be taken into consideration. The key focus of a realist approach is on "what works for whom, in what contexts, and how'. Realist evaluation is particularly useful when new interventions are being developed; when interventions are being considered for replication or scaling up; when programs are complex or are being introduced in complex settings; or when previous evaluations of programs have found mixed outcomes (Westhorp 2014).

The evaluation of Kids Together was also a shared activity; working collaboratively with Noah's Shoalhaven, a not for profit children's charity based in Nowra, New South Wales (NSW, Australia), and an expert Steering Committee, enabled the researcher team to gain a better understanding of the broader social, policy and organisational contexts in which the program had been developed and was being delivered. The evaluation had formative, process and summative elements. The key questions for the evaluation were:

1. What were the outcomes for individual children?

2. What was the impact on the early childhood educators and the Kids Together key workers delivering the program?

3. How effective was the transdisciplinary approach (training/coaching/modelling) in developing the knowledge, skills and attitudes necessary for ongoing implementation of the Kids Together model in an early childhood learning setting?

4. What was the broader social impact?

5. What are the prospects for the broader implementation of the model? 
The evaluation was conducted in three local government areas (Illawarra, Shellharbour and Kiama) in New South Wales over a 12 month period (January to December 2014) by a team of researchers from a regional Australian university. The sample of ECEC centres for the formative and process elements were chosen to reflect the socio-economic differences and geographic diversity of the region, the various service delivery groups and ethnicity. Qualitative data was collected from five of the 15 ECEC centres. An outcomes evaluation was based on quantitative data collected across all 15 centres. We assessed the wider impact of the program at the community and societal levels and the suitability of the program for broader implementation across multiple sites. Ethical approval for the research was obtained.

\subsection{Developing Logic Models}

While there is considerable variation in the way logic models are used by evaluators, including the degree of complexity in the logic model, the primary purpose of a logic model is to articulate the underlying assumptions about how the expected outcomes of a program will be reached in the short, medium and long term. The underlying theory or 'program theory' explains how the program works and how the components of the program work together, rather than what it is expected to achieve. According to Gugiu and RodriguezCampos (2007) a program theory has two purposes: to determine how inputs support activities to produce the desired outcomes; and secondly to form the basis of a theory driven evaluation. Therefore, a program theory, which includes a program's inputs, activities, and its short, medium and long term outcomes, becomes a useful tool to guide the evaluation (Weiss 1972).

The development of a logic model does not assume that a program is static or unchanging.

Community programs are usually dynamic and continually evolving; the logic model can be 
thought of as a 'snap shot' in time in relation to a program and can be revised over the course of a program's life. Program logic models are frequently developed as part of the design phase of a program to communicate the program elements demonstrate the 'logic' of how inputs and activities will contribute to the desired impact and outcomes. For established programs the logic model provides the basis for a shared understanding about how the program works, and informs the development of an evaluation framework, systematic data collection and reporting. Alternatively the logic model may be reviewed after an evaluation is completed to ensure that the program learns from the evaluation findings and adapts appropriately. This process is most effective when it is collaborative and done through involvement of key stakeholders (Yeatman, Quinsey et al. 2013).

\subsection{Developing the Kids Together Model}

The Kids Together logic model delivered a visual representation of the underlying rationale or logic of the program. It provided the basis for a shared understanding about how Kids Together worked, and informed the design of an evaluation framework, systematic data collection, data analysis and reporting (Helitzer, Hollis et al. 2010, Newton, Poon et al. 2013). The process of developing the logic model provided a structured way to explore the complexity of what the Kids Together program offered with those involved and enabled the research team to work collaboratively with key stakeholders to document a growing understanding of how the model was being implemented (Baxter, Killoran et al. 2010, Yeatman, Quinsey et al. 2013). As noted by Epstein and Klerman (2013) it was important to be realistic because models in the early implementation phase 'are rarely experienced in their ideal form'.

The Kids Together logic model was based on initial interviews with key stakeholders. A semi-structured interview protocol derived from the evaluation literature (Gugiu and 
Rodriguez-Campos 2007) was adopted in order to facilitate the development of the logic model. There were multiple discussions with Noah's Shoalhaven and with the Steering Committee to determine if the logic model clearly communicated the how and why of this program and if the expected outcomes were appropriate and feasible. This clarification with key stakeholders provided the basis for the development of an evaluation framework (including process, outcomes and impact) which aligned the key evaluation questions, indicators and available data. The resultant model provides a visual representation of the Kids Together model and its component parts and enabled a theory of change to be inferred. A 'theory of change' explains how the program activities are understood to produce the series of results that contribute to achieving the final intended impacts (Rogers 2014). It can be used to identify what data should be collected and how it should be analysed and is an important first step to undertaking an impact and outcomes evaluation.

The Kids Together logic model presented in Figure 1 reveals that the program operates in a complex environment with many factors likely to influence its success. It situates the program as being implemented in multiple and diverse early childhood education settings in a regional area of New South Wales, Australia. There are a number of assumptions underlying the program; an overarching assumption was Noah's Shoalhaven's strong commitment to inclusion of children with disabilities through early childhood centres. There were significant changes in the external environment over the course of the evaluation. The major policy change of the introduction of the NDIS was of course a major impetus for the program's development and implementation. There was considerable uncertainty around the timing and details of the implementation of the NDIS over the evaluation period, and a lack of detailed information about the transition arrangements for service providers, including funding arrangements. The Kids Together program also represented an expansion of the operations of 
Noah's Shoalhaven from the Shoalhaven region of NSW where it has successfully operated since 1981, into the Illawarra region where it had to establish new networks.

The inputs in this model identify all the major resources that are necessary for the program to operate. The Kids Together logic model identifies five types of inputs: funding; Noah's Shoalhaven staff time; program knowledge and resources; Kids Together key worker skills and expertise; and relationships with ECEC sector. The model identifies nine key activities and involves six types of participants. The model then links inputs, activities and participants to the short, medium and long term outcomes. The model uses colour to highlight the logical outcomes of the program activities corresponding to each participant group. Sometimes the relationships between the elements of a program are connected by a series of arrows that represent the causal links between elements. To avoid clutter an alternative method is to assign each element with a unique identification code and replace the arrows with the codes of the related elements (Rodriguez-Campos and Rincones-Gomez 2012). In the model below (Figure 1) the unique identification code is shown in bold prior to the element descriptor. The related elements are shown in plain text after the element descriptor (IN1, A1, P1, ST1, MT1, LT1 etc.).

\section{INSERT FIGURE 1}

\subsection{Assessment of the program theory}

As described previously, the program theory refers to how the program is meant to operate in order to achieve the desired outcomes, and how the component parts of the program fit together (Weiss 1972, Gugiu and Rodriguez-Campos 2007, Rogers and Funnell 2011). As Rogers and Funnell (2011) point out, it can be impossible to interpret evaluation results correctly in the absence of program theory. 
The assessment of the program theory underlying the Kids Together model took into account: the needs of the target population the program was intended to serve; the logic or plausibility of the model; comparison with research and practice; and assessment through preliminary observation (Rossi, Lipsey et al. 2004). The desired key outcomes in the Kids Together model were derived from best practice in the current literature, and were particularly influenced by Moore's recent work on inclusion in early childhood intervention (Moore 2012).

The assessment of the strength of the program theory was based on our understanding of current literature which strongly supported the importance of a key worker transdisciplinary approach to achieving the short and medium term outcomes which would in turn lead to achieving the broader goals of inclusion. The preliminary observation and assessment of the professional knowledge and skills of the key worker team provided further support for the soundness of the program theory.

The logic model offered the program developers the opportunity to reflect deeply on the Kids Together approach and discuss why implementation of the planned activities would lead to the stated outcomes (Helitzer, Hollis et al. 2010, Ziviani, Darlington et al. 2011, Newton, Poon et al. 2013). Once finalised the logic model was used to frame the analysis of data and determine which outcomes were achieved during the first year of implementation. A series of flexible data collection tools and instruments were developed accordingly.

\subsection{Data sources and stages}

The evaluation was undertaken using a staged approach over a 12 month period. The evaluation was conducted over three stages. In stage one the researchers were engaged in 
setting up the evaluation, establishing a relationship with the key personnel through site visits, establishing a steering committee with the inclusion of key policy and practitioner stakeholders, preparing applications for ethical review and preparing the program logic model, evaluation framework and data collection instruments.

The initial data collection period was conducted in the early stages of the program implementation (February- April). It focused on obtaining the views of all participants about their expectations and early experiences of the program. It included the first round of qualitative data collection.

The researchers established initial contact with each of the sites in February 2014. Noah's Shoalhaven provided the research team with the names of five directors of participating ECECCs. The choice of which of the centres to be included in the evaluation was based on convenience and centre readiness and willingness to participate and by negotiation between Noah's Shoalhaven and the ECEC centres. Noah's Shoalhaven facilitated the initial introduction to the directors at each of the sites. The directors then provided the researchers with an orientation to each of the services and facilitated opportunities for data collection with themselves, staff and parents/carers.

In Stage two the researchers, collected both qualitative and quantitative data. Qualitative data collected included: Observations recorded at site visits at the 5 ECEC centres in September; a second round of face to face interviews (parents/carers; Noah's staff; ECEC Centre directors) at the five ECEC centres in November/December; a second focus group with ECEC Centre staff.

Quantitative data collected included: a survey of ECECC staff across all 15 sites in December in order to assess the extent to which the program's short and long term impact on 
the individual children, families, teachers, educators and centres involved, and on the Noahs Shoalhaven staff team; and the collection and analysis of de-identified GAS scores for individual children, obtained from Noah's Shoalhaven.

Stage three of the evaluation involved an analysis of how the Kids Together model may be implemented in other settings. To assess the effectiveness of the Kids Together model across a wider range of settings and investigation was undertaken to assess the suitability of the program for broader implementation; and the organizational capacity and options for program expansion. This information is intended to assist in identifying what would be required to replicate and expand the program across other settings.

Fifty-five participants recruited to take part in stage one and the data collection for this stage took place from February to July 2014. Two-hundred and eleven participants recruited to take part in stage two and the data collection took place from September through to December 2014.

The following sources of qualitative and quantitative data were collected and analysed:

- Face to face semi-structured interviews with three types of participants: Noah's Shoalhaven staff; ECEC centre directors; parents/carers)

- Focus group discussions with ECEC Centre staff

- Observations undertaken during site visits at intervention sites.

- Program data collection from a thorough review of Noah's Shoalhaven program documentation, meeting notes, websites etc.

- Quantitative data collected from and end of year survey with ECEC centre staff.

- Analysis of results of individual child Goal Attainment Scale (GAS) scores.

\subsection{Data collection}


The evaluation utilised a mixed-method design using qualitative and quantitative data collection tools and instruments developed by the researchers. Program data was collected from existing documentation, including Noah's Shoalhaven annual reports, website and routinely collected administrative data. Data was collected on site from each of the 15 sites in which the program was implemented. The ECECs were chosen for diversity, both in terms of industry type and demographic characteristics: they included nine long day care centres and six community preschools (two of which also offered occasional care); independent and corporate centres which included children from Aboriginal and Torres Strait Islander and Culturally and Linguistically Diverse (CALD) backgrounds.

Qualitative data was collected from a total of 124 participants using face-to-face semistructured interviews, focus groups and observations. Two rounds of face-to-face interviews and focus groups took place, one round at the beginning and the other at the end of the implementation period. Evaluators interviewed parents/carers of children with disabilities/additional needs; directors of ECEC centres and Noah's Shoalhaven key workers and staff. Focus groups were conducted with educators at five of the ECEC centres. Observation sessions were undertaken and field notes collected at five of the ECEC centres, mid-way through the implementation period.

Quantitative data was obtained from two sources: a survey of 87 early childhood educators and Goal Attainment Scaling (GAS) reporting routinely collected by Noah's Shoalhaven from 61 individual children. Originally developed for mental health evaluation (Kiresuk and Sherman 1986), GAS has been widely used in allied health fields including rehabilitation (Turner-Stokes 2009) and professional education (Campbell, Novak et al. 2013). GAS was used by in the Kids Together program to facilitate goal setting and assess child outcomes in an inclusion program for children with disabilities or additional needs 
(Williams, O'Brien et al. 2016).

\section{INSERT TABLE 1}

\subsection{Data analysis}

Data analysis involved the 'triangulation' of data obtained from the different methodological approaches described above, both as a strategy for validation (Denzin 1978, Morse 1991, Flick, Kardorff et al. 2004), and as a method of obtaining complementary findings that strengthen the research results and contribute to theory and knowledge development (Morse 1991). It is also intended to capture a more 'holistic and contextual portrayal' (Jick 1979) of the effectiveness of the program.

All of the qualitative data collected was entered into NVivo Software (QSR International Pty Ltd 2012). A project was set up in the NVivo computer program and interview transcripts were imported into the program along with researcher notes and observations program data, documents and literature. The semi-structured interviews were auto coded to systematically organise the answers to each interview question. Each question was matched to the process objectives or one or more of the short and medium term outcomes identified in the logic model. The semi-structured interviews were also analysed thematically, using the key themes which emerged from the data, to enable a more grounded approach. Framework analysis (Ritchie and Spencer 1994, Ritchie, Spencer et al. 2003) was then applied to the data. Richie and Spencer, who first developed the framework approach to qualitative data analysis in the context of applied social policy research, describe the framework approach as 'a systematic process of sifting, charting and sorting material according to key issues and themes (Ritchie and Spencer 1994). In this research it involved the development of a matrix consisting of a series of columns (containing the questions or themes) and rows (containing the interview 
cases). The data in each resultant cell was systematically summarised, and then reduced, in order to analyse it by case and by theme, while retaining links to the original data. As noted by Gale, this provided a systematic way for the multi-disciplinary research team to manage and map the qualitative data (Gale, Heath et al. 2013) and provided transparency in the data analysis.

The statistical analysis of quantitative data from the survey data and GAS goals utilised Excel to generate charts and descriptive statistics. As the data are ordinal, medians are reported rather than means and standard deviations. To check for systematic differences in score distributions between two or more groups of respondents, non-parametric tests (MannWhitney U and Kruskal-Wallis) were conducted using SPSS. These tests do not assume data are normally distributed and test the null hypothesis that distributions of responses are the same across two or more categories of a grouping variable. Non-parametric (Spearman) correlations were also calculated, as these are appropriate for ordinal data. Responses to the open questions were entered into an Excel spreadsheet and a content analysis undertaken; this well-established method allowed the open questions to be synthesised using a systematic and replicable method of categorising and counting themes (Evans and FitzGerald 2002, DixonWoods, S Agarwal et al. 2005).

Data synthesis in the Kids Together evaluation was achieved by bringing together the results of each component of data analysis around the process, outcomes and impact objectives in order to address each of the evaluation questions in a meaningful way; this was done for both the qualitative and the survey data.

\section{Findings}

\subsection{Implementation}


Kids Together was successfully implemented as planned across 15 ECEC centres as well as in home and community settings over the 2014 school year, following a pilot conducted in one ECECC over one school term in the previous year. Overall, across the 15 centres a total of 76 children were supported by Kids Together key workers with a further 14 children receiving some form of consultation by Noah's Shoalhaven. GAS reports were completed for 61 children. Around 8\% of children in the cohort were from Aboriginal and Torres Strait Islander background and another $8 \%$ were from CALD backgrounds. Home visits were conducted to 30 families, while around 70 families were provided with additional information and resources. Community outreach activities were successfully conducted in a small number of community settings. A variety of training opportunities were provided to ECEC centres using strategies such as coaching, modelling, information sharing and formal training.

Overall, parents/carers, directors, and ECEC centre staff who participated in the Kids Together reported a high degree of satisfaction with the program. Parents/carers interviewed strongly supported the need for the Kids Together program operating in an ECEC centre setting. Of the ECEC centre staff surveyed, two thirds rated their levels of overall satisfaction with the Program as 'excellent' or 'very good'. Overwhelmingly, staff members appreciated having the Kids Together key workers available in the centres. Kids Together key workers were seen as an important resource, a source of information, consultation and advice. ECEC centre staff had the opportunity to learn new approaches and develop skills. ECEC centre staff valued the Kids Together key worker's contribution to addressing specific issues to facilitate inclusion of the target children.

The experience of implementing Kids Together for the first time across 15 centres provided an opportunity for valuable lessons to be learnt, including the importance of providing good information to all stakeholders at the start of the program and the need to monitor and 
manage the increased workload of the Noah's Shoalhaven team delivering Kids Together across multiple ECEC centres. Over the course of the year, the workload of the Kids Together key workers became more manageable as key workers became more familiar with the model and developed ways to refine administrative and reporting processes. They also became more skilled at achieving a balance between work in the ECEC centres and in the homes and community settings. Finding better ways to manage time and allocate workload allowed for the essential components of Kids Together to be successfully delivered at the ECEC centre, at home and in the community.

\subsection{Achievements}

Overall positive results were recorded for all participant groups across multiple environments. The main findings summarised below highlight the outcomes for the children, parents/ carers, ECEC centre staff, Noah's Shoalhaven as well as the broader community and societal level outcomes and impacts of the model.

\subsubsection{Outcomes for Children}

As outlined in the logic model Kids Together proposed that for children to have the skills they need to function well at home, the ECEC centre and in the community, they need to be provided with sufficient opportunities to practice and achieve their goals in a range of environments relevant to each child. The short and medium term outcomes would be expected to lead to children having the skills to function well at home, the ECEC centre and in the community, and assist in their smooth transition to school for children.

The evaluation provided both quantitative and qualitative evidence to support that outcomes for children were achieved. Analysis of qualitative data indicated that children were able to build skills, specifically tailored to their individual needs, by getting multiple opportunities to 
practice these skills across a variety of settings. As the analysis of individual Goal Attainment Scoring (GAS) reports indicates in Term 4 a majority of children had reached or exceeded the individual goals that were set. A fuller discussion of the GAS results is provided in a separate paper. (Authors, forthcoming)

\subsubsection{Outcomes for Parents/Carers}

Kids Together set out to assist parents/carers by providing more support, build confidence and provide opportunities to learn and practice new skills. The longer term aspiration in relation to parents/carers was to ensure they had skills to support their children through school. The evaluation process collected evidence that both short and medium term outcomes were achieved during 2014.

The interviews provided overwhelming support for the program from parents/carers whose experience with Kids Together was very positive. Approximately half of the families participating in the Kids Together program received a home visit from a key worker. Home visits enabled the Kids Together team to work closely with families, to assist parents to develop skills and help them work better with external therapists, as evidenced in the following quotations from parents:

I think all of the [ECECC] staff are really interested and invested in where she's gotthey go out of their way to make sensory resources and things like that, they go out of their way a bit for her, and they're learning, I feel like they're learning about sensory defensiveness, where it was probably not there earlier, if that makes sense. Parent 
(My son) is now able to tell them that he needs something and they've got the resources there to be able to talk to - to deal with him. (Noah's key worker) taught the centrekey teachers - on how to deal with it. Parent

Parents/carers indicated in interviews that they built knowledge and skills to support their child over the course of the year. Strategies learnt related to a variety of different areas such as: fine motor skills; language; sensory; play and wait; speech; behaviour (accidents and biting); praise and acknowledgement; limiting attention for non-compliant behaviour and for some becoming a patient parent. Parents/carers said that they found tools and strategies provided to be easy to understand and implement at both the ECEC centre and at home.

I think it has been a Godsend. It has really helped (my son) at school. It's helped his teachers know how to deal with him. (Kids Together key worker) has helped me know how to deal with him and that has just been invaluable. I think it's a great program.

\section{Parent}

Over time parents developed greater confidence to implement and regularly practice the support strategies they learnt both at home and at the ECEC centre. Kids Together also provided practical support and information to all parents/carers in relation to their child's transition to school. Parents/carers expressed more confidence in making the best decisions about their child as they transitioned to school. Additionally, parents/carers supported the expansion of Kids Together to more ECEC centres and felt that it would be helpful to offer Kids Together in primary schools.

\subsubsection{Outcomes for Early Childhood Education Centres}


Kids Together aimed to achieve sustained inclusive practice across all areas of the ECEC centre in the long term, through a series of short and medium term outcomes focused on the ECEC centre staff, the ECEC centre environment and on sustaining changes. In the short term they aimed to enhance staff knowledge around inclusive practices, improve their skills through coaching and training and increase their access to relevant information and resources. In the medium term the model aims to increase staff skills and confidence in implementing the inclusive strategies learned; to achieve good inter-professional collaborative learning between Kids Together key workers and staff; and to improve staff relations with both children and parents/carers.

In their survey responses, ECEC centre staff members were highly positive about the levels of support they received from Kids Together key workers and their own centres, and strongly endorsed items asking about their levels of confidence, skills and access to information following the intervention. Nine out of ten respondents reported that Kids Together had led to better ways of working with children who have disabilities/additional needs.

\section{INSERT FIGURE 2}

Figure 2 shows respondents' ratings of their knowledge, skills and confidence following the implementation of the Kids Together program at their centre. Highly enthusiastic responses that is, ratings of 'excellent' or 'very good' - were given by more than half the staff in relation to their training, levels of confidence and access to information. Forty-two respondents $(58.3 \%)$ said their skills for including children with disabilities in a mainstream early childhood setting were now 'excellent' or 'very good'. Levels of satisfaction were rated as 'excellent' or 'very good' by two thirds of respondents. However, six respondents $(8.3 \%)$ rated their satisfaction as only 'fair' and a further two declined to answer this question. 
Not all respondents were highly positive. Ten staff members (13.9\%) rated their knowledge as only 'fair', and nine (12.5\%) rated their confidence and/or opportunities to practice skills as only 'fair'. Fourteen respondents (19.4\%) rated their training as 'fair' or 'poor' and thirteen respondents $(18.0 \%)$ reported that their access to resources was 'fair' or 'poor'.

Both the staff survey and the qualitative data confirmed the level of knowledge acquisition which occurred as part of the Kids Together program. ECEC centre staff learned new strategies, skills and/or techniques, gained knowledge and achieved personal development, such as confidence, patience and opportunities for self-reflection.

I feel I have learnt more this year than my whole career ECEC centre deputy director

You're sharing your knowledge with somebody who has got more knowledge and then putting it all together and figuring out what's best for that child and that's why I think this works. ECEC centre staff

I had not had any specific training on working with children with additional needs, so everything that [Kids Together key worker] has given us has been invaluable. ECEC centre staff

The coaching and training provided opportunities for inter-professional collaborative learning between the Kids Together key workers and ECEC centre staff. The majority of staff members surveyed 'strongly agreed' or 'agreed' that they felt well supported by the program and almost four in five reported improved quality of interactions with children at their centres. 
There was a notable increase in ECEC centre staff access to useful and relevant information and resources especially for children with additional needs connected to sensory processing disorder. This was reinforced by the availability of Kids Together key workers on a regular basis (weekly visits) to assist ECEC centre staff in accessing information and resources and to answer questions as they arose. This access to the Kids Together key workers was highly appreciated by the majority of the ECEC centres.

Results from the survey gave cause for optimism about the prospects for sustaining the program and implementing it more widely. In general, ECEC centre staff members believed the program could be sustained and acknowledged that policies and procedures were in place to support its continuation in their own centres. The vast majority said that they would like to see Kids Together continue and operate in other ECEC centres.

In the interviews, ECEC centre directors were overwhelmingly supportive of the program and shared how they had observed changes at their ECEC centres where staff had increased skills and confidence which had them taking ownership of the ongoing application.

The staff have taken ownership of that knowledge, it's just a logical progression to apply to new children, it's the existing children that are there, whether the key worker is still part of the service or not. It'd be lovely to be able to have that professional input and that continuing forever, but I mean, I think what the staff are learning is, I mean, it's a logical progression. ECEC centre director

They provided examples of how the Kids Together key worker consistently helped ECEC centre staff to understand strategies and techniques to support the children from the different 
therapy perspectives. The majority of ECEC centre directors believed the changes achieved during the implementation period could be sustained in their Centre over time.

\subsubsection{Outcomes for Noah's Shoalhaven}

The analysis of qualitative interviews and field notes from mid-year observations indicated that Kids Together key workers were highly successful in achieving the desired short term outcomes shown in the logic model: effective work by Kids Together key workers with children within the ECEC centre, home and community; and effective transdisciplinary team processes developed across all ECEC centres. Findings indicated that these could be expected to lead to effective peer learning between key workers in the transdisciplinary team, in the medium term.

Key workers were particularly successful in developing ways of working within the wide variety of ECEC centre cultures and different levels of ECEC centre staff skills and motivation. They felt that they had empowered ECEC centre staff, built knowledge, skills and confidence; at times they stepped back and allowed time for ECEC centre staff to reflect, adapt and take ownership of the strategies.

(ECECC staff) felt much more empowered because they had the education, they had the knowledge. By giving them the opportunity to increase their skills and their confidence they were more likely to develop an ability to look at things through a slightly different lens. The more informed lens equated to a more robust and supported way to true inclusion and not just an add-on bit. Kids Together key worker 
It meant that we were able to support and offer our variety of expertise over a larger number of ECEC centres. The team's expertise is funnelled into supporting each of us which was an efficient way of getting the child with additional needs all the appropriate support required. Kids Together key worker

\subsubsection{Community level outcomes and societal impacts}

Overall parents reported varying levels of confidence in their preparedness to include their child in a broader range of community and social settings. All participants agreed that having the opportunities to practice new skills in the community was an important and necessary element of Kids Together. Several parents/carers provided examples of having successfully used resources and strategies learnt through Kids Together with their children in community environments, such as shopping centres and parks.

So when I go to the shopping centre now I make sure I have a backpack so he's got his headphones in it, he's got things to blow. We took the snake [weighted sensory resource] the other day just to try and-yeah. So it is constant. Parent

At the park. He - at the beginning of the year he would sit back and just play with his sister and that's it. But now he'll go to the park and he'll be playing with everyone else except his sister. So - but his confidence is a lot better. Parent

The meaningful participation of children and families as valued members of society is a long term aspirational goal of Kids Together, a goal which is consistent with the disability reform policies within Australia. Although a full report on progress towards this outcome was beyond the scope of the evaluation primarily because it was conducted after the relatively 
short implementation period of 12 months, the evaluation identified many examples of short and medium term program achievement at the individual and organisational levels.

\section{Discussion and conclusion}

The evaluation documented the successful implementation of the Kids Together model by Noah's Shoalhaven in a 'real world' setting across a diverse range of early childhood education settings within an urban/regional setting in NSW. The program identified, assessed and supported children with disabilities/additional needs and achieved significant outcomes for all participant groups. The trial across 15 ECEC centres provided many opportunities for the Kids Together key worker team to gain experience in the model and learn valuable lessons which will inform future implementation.

Building strong support relationships between key workers, ECEC centre staff and parents was seen as an underlying pillar of the model from the outset. The model of delivery in which the relationship building starts in the ECEC centre had the effect of simplifying service delivery for the parents/carers. Parents/carers observed increased capacity in the ECEC centres as well as positive changes in their children. Parents were enthusiastic about the program from the outset, and remained so throughout the year; the way the relationships were built up over time clearly made parents/carers feel supported and enabled them to better understand their children's conditions and how their children could benefit from the program.

From an ECEC centre perspective, having the model based at the centre greatly facilitated the coordination of therapies. The model provided processes for problem solving and was flexible enough to achieve continuous improvement as the team gained experience in working together across various settings. A significant achievement was the establishment 
and further development of new ways of working by Noah's Shoalhaven using a transdisciplinary framework reported in the literature to be best practice (Moore 2012).

Inter-professional collaborative learning between the Kids Together key workers and ECEC centre staff was developed through coaching and training. Kids Together key workers adapted their approach to coaching to suit the different skill levels and training qualifications of ECEC centre staff. Successful coaching was enhanced by Kids Together key workers being regarded as 'part of the team' and having consistent ECEC centre staff and shared goals for the children. Kids Together key workers worked on the floor with the child and this enabled staff to observe and then try strategies themselves. Coaching worked best when the ECEC centre staff were well supported by their ECEC centre directors and there was flexibility around the program. Kids Together key workers observed that ECEC centre staff became more engaged, and increased in skills and confidence over time. Kids Together key workers recognized that their own coaching skills and understanding of the technique improved over time.

The results of the evaluation demonstrate that there was a high level of overall success across all centres; however the similarities and differences reported about the way the program worked across the 15 ECEC centres are worth noting. In general, staff members believed the program could be sustained and acknowledged that policies and procedures were in place to support its continuation in their own centres.

The results of the qualitative data provided further insight into how the program worked somewhat differently across the centres. Notably, the model worked best where there was strong leadership from the centre directors and deputy directors and where directors allocated specific staff time to working with Kids Together tasks. In the centres where, for a variety of 
reasons, there was not a strong staff allocation to Kids Together, staff were not always available to work alongside the Kids Together key workers and therefore potential for 'teachable moments' was somewhat reduced.

Finding the right communication strategies for each of the centres was a critical success factor. This meant not only adapting to the culture of early childhood, but also adapting to the needs and preferences of the particular centres and coming up with new methods of communication. Particularly important was the need to keep all stakeholders well informed and updated on the progress and changing needs of children. This was a challenge which was solved in a variety of innovative ways by the Kids Together key workers, including various forms of information technology, to ensure all parties were informed of developments. The success of these strategies also appeared to depend on the type of centre. The program worked best where Kids Together key workers were able to adapt communication strategies to suit the particular centre.

\section{Conclusion}

The evaluation provided evidence that the Kids Together model met the identified need for a multi-faceted key worker transdisciplinary inclusion model based within the early childhood education setting. The model filled an unmet demand for service delivery to children with disabilities/additional needs and the families who support them. The timing of the implementation was important as it came at critical point in regard to the growing awareness of inclusion through the introduction of the NDIS, greater media exposure to the issue, and an apparent increase in diagnosed cases of conditions such as autism as well as behavioural disorders. This meant that parents, carers and ECEC centres staff were all open to the program and welcomed the introduction. 
Underlying its successful implementation is that the program came at no cost either to the ECEC centres or to parents. The ECEC centre directors were committed and welcomed the program from the outset. Their enthusiasm for the program continued over the course of the year as it became evident that the style of the Kids Together key workers complemented the educationalists' approach to early childhood education as it worked within the philosophic principles of the Early Learning Framework (Australian Government Department of Education Employment and Workplace Relations 2009).

In summary, the evidence from the evaluation strongly suggested that Kids Together was a highly effective and innovative model for supporting the inclusion of children with disabilities/ additional needs in an early childhood education and care setting. The following points were found to be key strengths of the model:

- The key worker model simplified the coordination of therapies at the ECEC centre level, built the capacity of ECEC centres and provided benefits for children and families.

- The Kids Together key worker was the link to all services. Parents/ carers only had to deal with one person. This was particularly important for families from lower socio-economic and Indigenous backgrounds.

- The Kids Together key worker managed access to other services. This avoided the problem of multiple therapists having to enter the ECEC centre.

- The model worked across all of the environments where children with disabilities/additional needs lived and played: ECEC centre, home and community settings.

- Children benefitted from the multiple opportunities to practise the goals set for them across several environments. 
- Parents and carers were supported by regular communication and advice about their child's progress.

- ECEC centre staff capacity was built through multiple training opportunities using strategies such as coaching, modelling, information sharing and formal training.

Kids Together was developed during a period of radical policy change which put pressure on existing service providers in the disability sector in Australia, demanding innovative ways of delivering services to people with disabilities. Moreover, the inclusion of children with disabilities in the early childhood education sector is also a relatively new practice within Australia, with few evaluated programs available to guide program development. The use of a logic model in this context provided the program developers and the evaluation team with a conceptual framework which facilitated a shared understanding of the relationship between available resources, activities across various domains and the extent to which desired outcomes were and could be achieved in the short, medium and long term.

The logic model helped to show how a coordinated and collaborative approached can work across multiple environments. Working with one key person supported by a team of experienced healthcare professionals proved to be extremely valuable for ECEC centres and individual teachers. This approach also assisted parents and carers in trying to navigate the best way to support their children with disabilities and enabled children to meet their individual goals with the potential of improving their meaningful engagement in society. Based on the results of the evaluation the future prospects for the broader implementation of the model across a wider range of settings were very positive.

\section{Lesson Learned}

There were three key lessons learned by the evaluators when reflecting on the use of the logic model. Firstly, the flexibility of the model was helpful when undertaking a realist evaluation approach and testing assumptions by evaluators with program developers and relevant 
stakeholders. Secondly, the logic model supported a collaborative approach in building a shared understanding throughout the evaluation process. Thirdly, the model provided a structured way of analysing and reporting on the mixed method evaluation findings.

\section{Acknowledgements}

We acknowledge the Aboriginal traditional owners of the Illawarra and Shoalhaven regions and pay our respects to elders past and present. We thank all those who participated in the evaluation for sharing their valuable time and insights. In particular we thank the parents and carers who agreed to participate in interviews, and the directors and ECEC Centre staff. We acknowledge that the evaluation has been a collaborative effort between the researchers and Noah's Shoalhaven. The research team would also like to thank the members of the Steering Committee for their advice in guiding the evaluation and for the ongoing efforts towards the social inclusion of children with additional needs. Funding for the evaluation was provided to Noah's Shoalhaven from the NSW Department of Family and Community Services. We acknowledge and thank the NSW Department of Family and Community Services for supporting this work.

\section{References}

Australian Government Department of Education Employment and Workplace Relations (2009). Belonging, Being and Becoming: The Early Years Learning Framework for Australia, Commonwealth of Australia.

Author and Author (2015). Evaluation of the Noah's Shoalhaven Kids Together Program. Wollongong, Australian Health Services Research Institute, University of Wollongong. Baxter, S., A. Killoran, M. P. Kelly and E. Goyder (2010). "Synthesizing diverse evidence: the use of primary qualitattive data analysis methods and logic models in public health reviews." Public Health 124: 99-106.

Bortolli, T. D. and A. Bundy (2015). Evidence for inclusion-based interventions for children aged 0-8 years and their families: A literature review. 26th ECIA (Early Childhood Intervention Australia) NSW Conference: Ready, Set, Let's Go Together. Sydney Brighton Beach. 
Campbell, L., I. Novak, S. Mclntyre and S. Lord (2013). "A KT intervention including the evidence alert system to improve clinician's evidence-based practice behaviour-a cluster randomized controlled trial." Implementation Science 8(1): 15.

Denzin, N. K. (1978). The Research Act. New York, McGraw-Hill.

Dew, A., T. D. Bortoli, J. Brentnall and A. Bundy (2014). Strengthening Supports for Children $0-8$ years and their Families: A Literature Review. Sydney, Faculty of Health Sciences, The University of Sydney.

Dixon-Woods, M., S Agarwal, D Jones, B. Young and A. Sutton (2005). "Synthesising qualitative and quantitative evidence: a review of possible methods." Journal of health services research and policy 10: 45-53B.

Epstein, D. and J. Klerman (2013). "When is a Program Ready for Rigorous Impact Evaluation? The Role of a Falsifiable Logic Model." Evaluation Review 2013(5): 375-401. Evans, D. and M. FitzGerald (2002). "Reasons for physically restraining patients and residents: a systematic review and content analysis." International Journal of Nursing Studies 39(7): 735-743.

Flick, U., E. v. Kardorff and I. Steinke, Eds. (2004). A companion to qualitative research. . London; California, Thousand Oaks; Sage Publications.

Gale, N. K., G. Heath, E. Cameron, S. Rashid and S. Redwood (2013). "Using the framework method for the analysis of qualitative data in multi-disciplinary health research." BMC Medical Research Methodology 13: 117.

Gugiu, P. C. and L. Rodriguez-Campos (2007). "Semi-structured interview protocol for constructing logic models." Evaluation and Program Planning 30(4): 339-350.

Helitzer, D., C. Hollis, B. Urquieta de Hernandez, M. Sanders, S. Roybal and I. Van Deusen (2010). "Evaluation for community-based programs: The integration of logic models and factor analysis." Evaluation and Program Planning 33: 223-233.

Howard, A., T. Blakemore, L. Johnston, D. Taylor and R. Dibley (2015). "'I'm not really sure but I hope it's better': early thoughts of parents and carers in a regional trial site for the Australian National Disability Insurance Scheme." Disability and Society 30(9): 1365-1381. Jick, T. D. (1979). "Mixing Qualitative and Quantitative Methods: Triangulation in Action." Administrative Science Quarterly 24(4).

Kiresuk, T. and R. Sherman (1986). "Goal attainment scaling: A general method for evaluating comprehensive community mental health programs " Community Mental Health Journal 4: 443-453.

Lee, F., A. Yeung, D. Tracey and K. Barker (2015). "Inclusion of Children With Special Needs in Early Childhood Education: What Teacher Characteristics Matter." Topics in Early Childhood Special Education 35(2): 79-88.

Moore, T. (2012). Rethinking early childhood intervention services: Implications for policy and practice

Pauline McGregor Memorial Address presented at the 10th Biennial National Conference of Early Childhood Intervention Australia, and the 1st Asia-Pacific Early Childhood Intervention Conference, Perth, Western Australia, 9th August 2012, Murdoch Childrens Research Institute and The Royal Children's Hospital Centre for Community Child Health. Morse, J. M. (1991). "Approaches to Qualitative-Quantitative Methodological Triangulation." Nursing Research 40(2): 120-123.

National Disability Insurance Scheme. (2014). "National Disability Insurance Scheme." Retrieved 17 June, 2016, from http://www.ndis.gov.au/document/what-national-disabilityinsuran.

Newton, X., R. Poon, N. Nunes and E. Stone (2013). "Research on teacher education programs: Logic model approach." Evaluation and Program Planning 36: 88-96. 
Odom, S. L. (2000). "Preschool inclusion: what we know and where we go from here." Topics in Early Childhood Special Education 20(1): 20-27.

Odom, S. L., Buysse, V., \& Soukakou, E. (2011). "Inclusion for young children with disabilities: a quarter century of research perspectives." Journal of Early Intervention 33: 344-356. Pawson, R. and N. Tilley (1997). Realistic Evaluation, Sage.

Pawson, R. and R. Tilley (2004). Realist Evaluation. Accessed on 1 November 2016 from www.communitymatters.com.au/RE chapter.pdf.

QSR International Pty Ltd (2012). NVivo qualitative data analysis software; Version 10. Ritchie, J. and E. Spencer (1994). Qualitative data analysis for applied policy research. Analyzing qualitative data. B. R. Bryman A. London, Routledge: 173-194.

Ritchie, J., L. Spencer and W. O'Connor, Eds. (2003). Carrying out Qualitative Analysis. Qualitative Research Practice. A Guide for Social Science Students and Researchers. London, SAGE Publications Ltd.

Rodriguez-Campos, L. and R. Rincones-Gomez (2012). Collaborative Evaluations Step-byStep, Second Edition. Stanford, Stanford University Press.

Rogers, P. (2014). Theory of Change, Methodological Briefs: Impact Evaluation 2. Florence, UNICEF Office of Research.

Rogers, P. J. and S. C. Funnell (2011). The essence of program theory. Purposeful program theory. P. J. Rogers and S. C. Funnell, Wiley.

Rossi, P., W. Lipsey and H. Freeman (2004). Evaluation: A systematic approach. Thousand Oaks, CA, Sage.

Sainato, D., R. Morrison, S. Jung, J. Axe and P. Nixon (2015). "A Comprehensive Inclusion Program for Kindergarton Children With Autism Spectrum Disorder." Journal of Early Intervention 37(3): 208-225.

Tanner, K., K. Cook and K. Clapham (2013). Inclusion of children with disabilities in early childhood education: A literature review. Wollongong, NSW, Centre for Health Service Development, Australian Health Services Research Institute, University of Wollongong. Turner-Stokes, L. (2009) "Goal attainment scaling (GAS) in rehabilitation: a practical guide." Clinical Rehabilitation 23, 362-370 DOI: 10.1177/0269215508101742.

Underwood, K., A. Valeo and R. Wood (2012). "Understanding inclusive early childhood education: a capability approach." Contemporary Issues in Early Childhood 13(290-299). W.K. Kellogg Foundation (2004). Logic Model Development Guide. Michigan, W.K. Kellogg Foundation. Available online at http://ww2.wkkf.org/Default.aspx?tabid=90\&CID=281\&ltemID=2810002\& NID=2820002\& LanguagelD=0. Accessed 15 April 2011.

Weiss, C. (1972). Evaluation. Upper Saddle River, NJ, Prentice-Hall.

Westhorp, G. (2014). Realist Impact Evaluation: An Introduction. A Methods Lab Publication Accessed 1 November 2016 from https://www.odi.org/publications/8716-methods-labrealist-impact-evaluation-introduction

Williams, K., G. O'Brien, M. Sutherland and C. Manning (2016). Use of Goal Attainment Scaling (GAS) in the Evaluation of the Kids Together Inclusion Program in Early Childhood Learning Environments. Paper presented at Early Childhood Intervention National Conference, Choices that matter: $\mathrm{ECl}$ in a new era. Melbourne, Victoria.

Yeatman, H., K. Quinsey, J. Dawber, W. Nielsen, D. Condon-Paoloni, S. Eckermann, D. Morris, P. Grootemaat and D. Fildes (2013). Stephanie Alexander Kitchen Garden National Program Evaluation: Final Report, Centre for Health Service Development, Australian Health Services Research Institute, University of Wollongong. 
Ziviani, J., Y. Darlington, R. Feeney and B. Head (2011). "From policy to practice: A program logic approach to describing the implementation of early intervention services for children with physical disability." Evaluation and Program Planning 34: 60-68. 
Fig 1. Logic Model

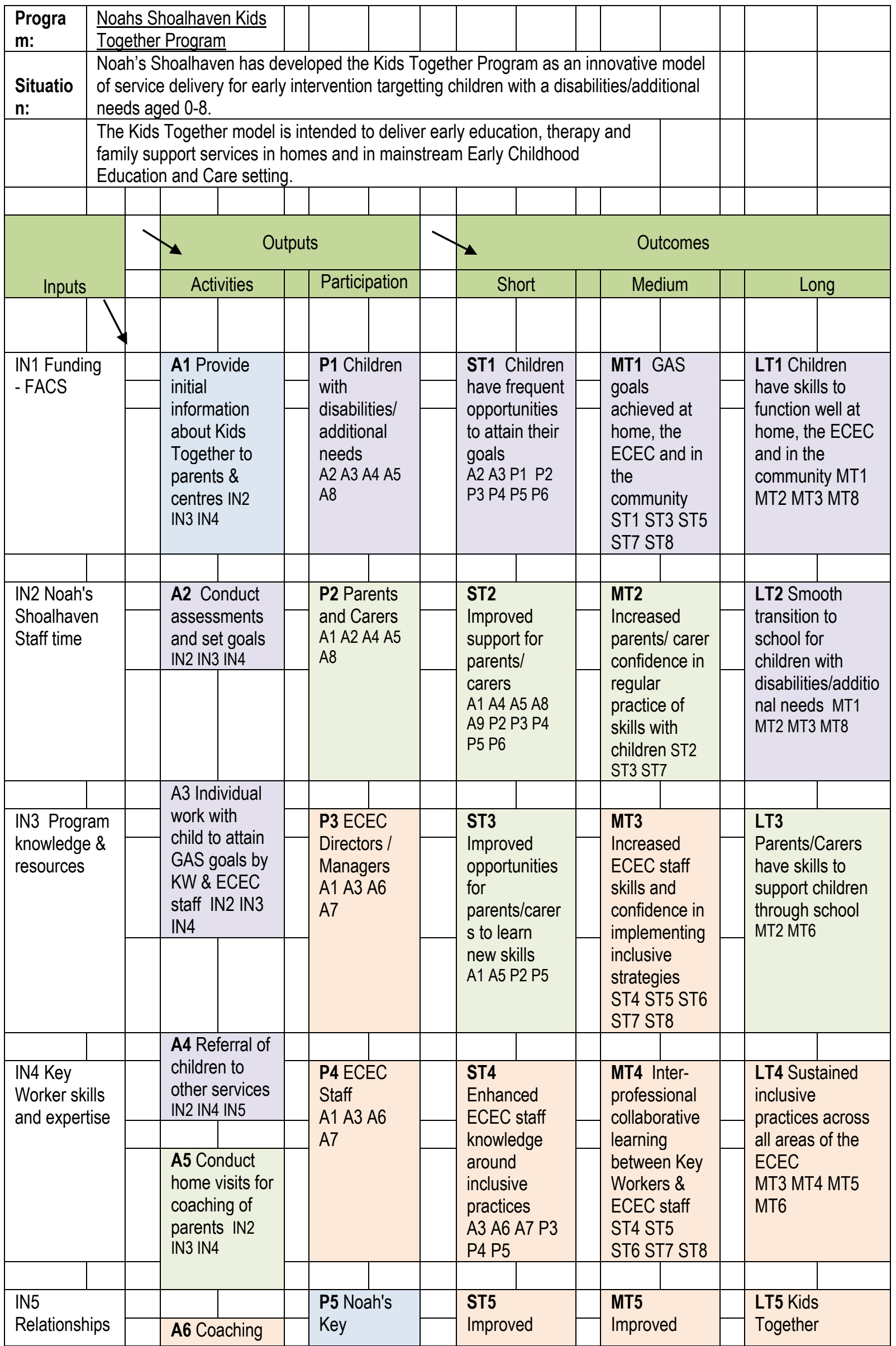




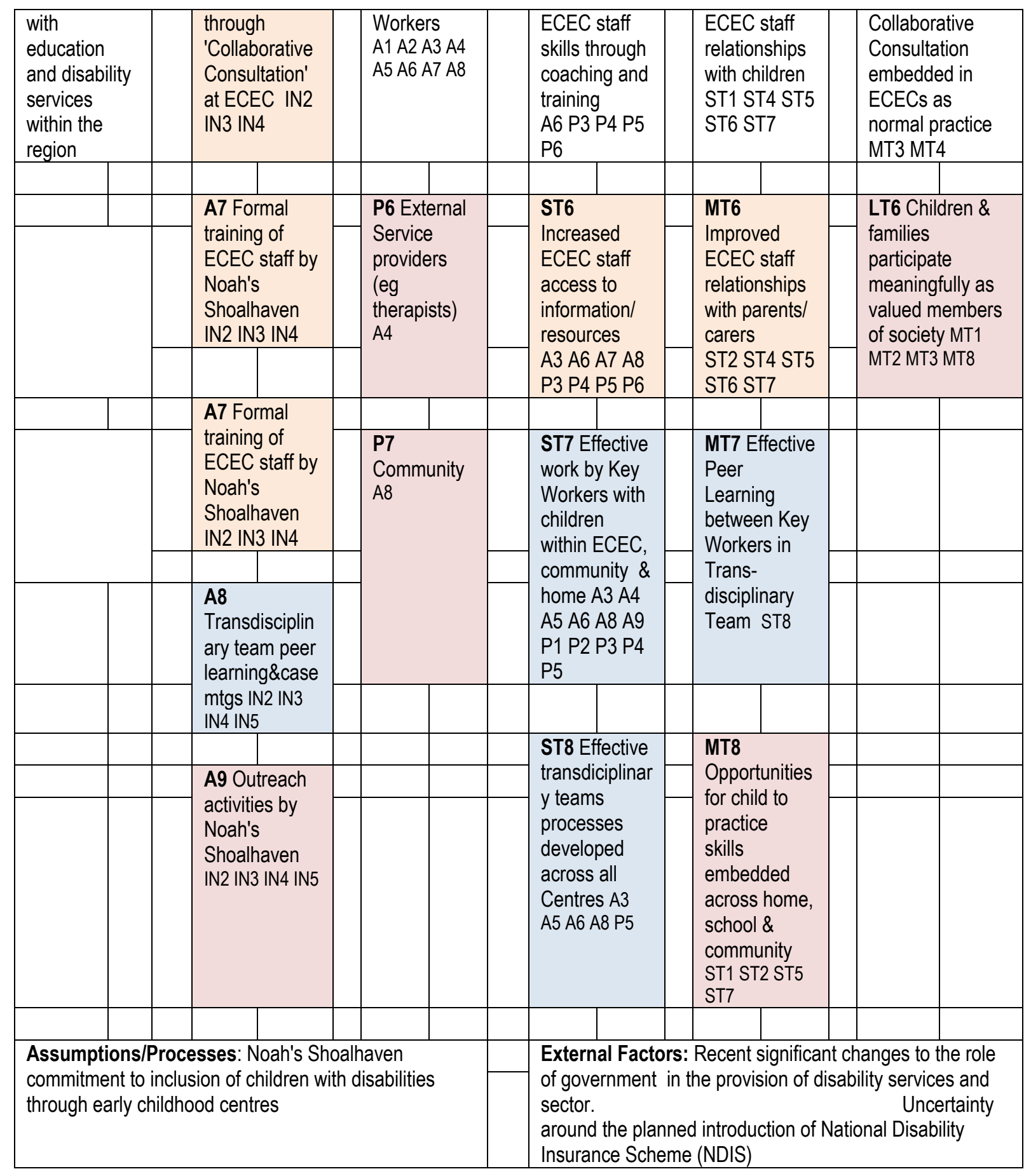


Figure 2 Staff members' knowledge, skills and confidence following the program

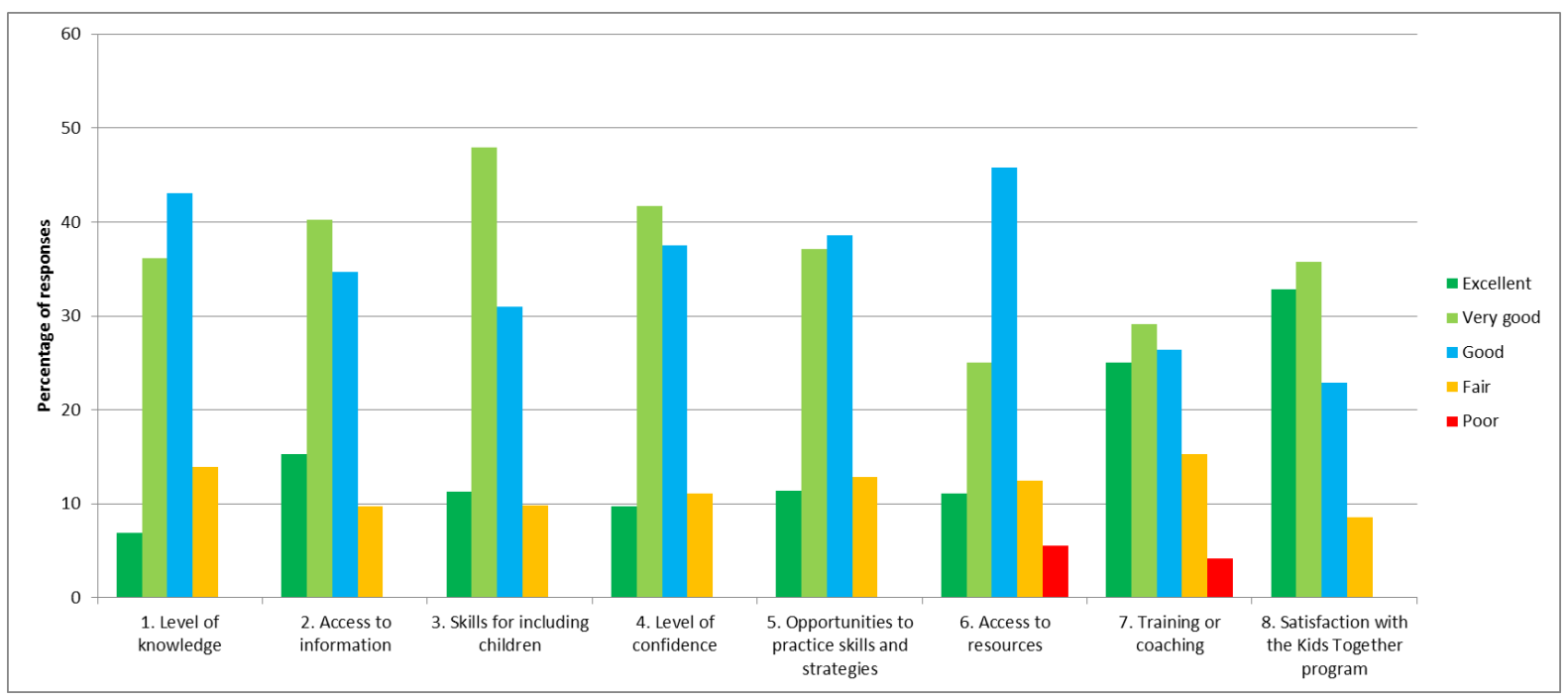


Table 1 Types of data collected

\begin{tabular}{|c|c|c|c|c|}
\hline \multirow[t]{2}{*}{ Type of data collected } & \multicolumn{2}{|c|}{ (Feb to July 2014) } & \multicolumn{2}{|c|}{ (Aug to Dec 2014) } \\
\hline & $\begin{array}{l}\text { Number of } \\
\text { interviews/ } \\
\text { FGDs/Survey } \\
\text { sessions }\end{array}$ & $\begin{array}{l}\text { Total } \\
\text { number of } \\
\text { participants } \\
\text { in research } \\
\text { activities }\end{array}$ & $\begin{array}{l}\text { Number of } \\
\text { interviews/ } \\
\text { FGDs/Survey } \\
\text { sessions }\end{array}$ & $\begin{array}{l}\text { Total } \\
\text { number of } \\
\text { participants } \\
\text { in research } \\
\text { activities }\end{array}$ \\
\hline $\begin{array}{lll}\text { Semi-structured } & \text { interviews } & - \\
\text { Directors[1] } & & \end{array}$ & 5 & 8 & 5 & 7 \\
\hline $\begin{array}{l}\text { Semi-structured interviews - NS } \\
\text { Kids Together team }\end{array}$ & 8 & 8 & 9 & 9 \\
\hline $\begin{array}{lll}\text { Semi-structured interviews } & - \\
\text { Parents/Carers } & \end{array}$ & 11 & 12 & 10 & 11 \\
\hline $\begin{array}{l}\text { Focus group discussion }- \\
\text { ECECC } \text { staff[2] }\end{array}$ & 5 FGDs & 35 & 5 FGDs & 29 \\
\hline $\begin{array}{l}\text { Participant observation at } \\
\text { ECECCs }\end{array}$ & \multicolumn{2}{|l|}{ Stage two only } & 5 sessions & 48 \\
\hline $\begin{array}{l}\text { Survey of ECECC staff (includes } \\
\text { directors) }\end{array}$ & \multicolumn{2}{|l|}{ Stage two only } & 15 ECECCs & 87 \\
\hline \multirow[t]{2}{*}{$\begin{array}{l}\text { Program data collected on child } \\
\text { development and progress }\end{array}$} & \multicolumn{2}{|c|}{ Stage two only } & 75 & 75 \\
\hline & & 63 & & 266 \\
\hline
\end{tabular}

[1] Directors were involved in both semi-structured interviews and focus group discussions 